\title{
Modern aspects of construction of information microwave therapy devices
}

\author{
Stanislav N. Darovskih ${ }^{1}$, Evgenij P. Popechitelev ${ }^{2}$, Nadezhda V. Vdovina ${ }^{1}$, Ivan V. Novikov ${ }^{1}$ \\ ${ }^{1}$ South Ural State University, Chelyabinsk, Russian; nadzh@yandex.ru \\ ${ }^{2}$ Saint Petersburg Electrotechnical University "LETI”, Saint Petersburg, Russian; eugeny p@mail.ru
}

Received 17 September 2013; revised 17 October 2013; accepted 24 October 2013

Copyright (C) 2013 Stanislav N. Darovskih et al. This is an open access article distributed under the Creative Commons Attribution License, which permits unrestricted use, distribution, and reproduction in any medium, provided the original work is properly cited. In accordance of the Creative Commons Attribution License all Copyrights (C) 2013 are reserved for SCIRP and the owner of the intellectual property Stanislav N. Darovskih et al. All Copyright (C) 2013 are guarded by law and by SCIRP as a guardian.

\begin{abstract}
This article is devoted to presenting modern aspects of building information microwave therapy devices. And it presents an analysis of the existing problems of the organism homeostasis control by means of electromagnetic radiation. The new principles of synthesis of operating influences are formulated. On the basis of the thermodynamic approach is justified as a leading role modeling the space microwave background to restore disturbed homeostasis of the organism. The general patterns of development of the pathological process and possible mechanisms of corrective action of natural electromagnetic background are described. The implementation of the approach offered by the authors to constructing device information physiotherapy allowed creating high-performance hardware and software means for the prevention and treatment of a wide range of human diseases.
\end{abstract}

Keywords: Information Microwave Therapy; Homeostasis; Electromagnetic Pollution; Relict Radiation; Electromagnetic Radiation

\section{INTRODUCTION}

An important feature of the current state of the environment is a growing impact of electromagnetic radiations of anthropogenic origin on living objects.

At all stages of the evolution of organisms, natural electromagnetic background played an information managing role, which is still poorly studied as for its mechanisms, in maintaining the ability of biological systems to resist changes in the external and internal environment and preserve the relative dynamic constancy of its struc- ture and properties.

Electromagnetic pollution caused by anthropogenic factor and called electromagnetic smog, now practically eliminates the controlling role of natural electromagnetic factor in maintaining homeostasis of the organisms (Figure 1). It creates the conditions for the emergence of unpredictable adverse effects in medical and biological terms. This is confirmed by numerous studies carried out in Russia and abroad [1].

At the same time, we can't recognize an existing medical practice of using low-intensity electromagnetic radiation of artificial origin for therapeutic purposes. For decades of their use in health-care facilities in several countries (Russia, Ukraine, etc.), numerous evidences of the therapeutic effect have been received [2].

But the paradoxical nature of this effect is connected with the fact that it is achieved by the use of EMR with the time-frequency structure similar to that of identical emission sources of electromagnetic pollution. The choice of "therapeutic radiation" is made without considering of the controlling role of electromagnetic factor of exogenous origin for natural organisms and current state of the electromagnetic pollution.

Such consistency and isolation from the external conditions when assessing the role of electromagnetic radiation for organisms indicate the presence of conceptual problems underlying the understanding of the mechanisms of its positive and negative effects on their living objects.

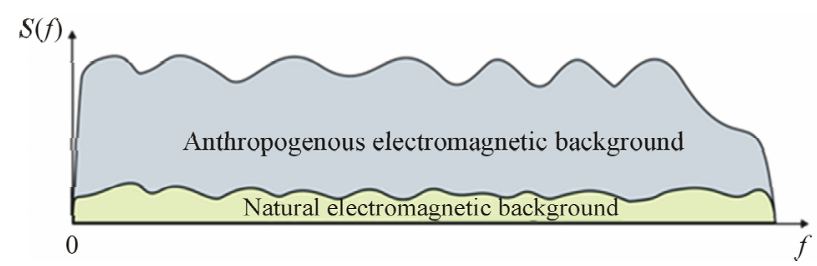

Figure 1. Illustration of a ratio of intensity anthropogenous and natural electromagnetic backgrounds. 
Resolution of these problems is related to new approaches in the evaluation of the mechanisms of interaction of organisms with electromagnetic radiation and the development of new principles of high-performance hardware for the prevention and treatment of a wide range of human diseases based on it. All the aforesaid cases determine the relevance of modern aspects of the use of microwave range electromagnetic radiation to restore disturbed homeostasis of the organism.

\section{THE PROBLEMS OF INFORMATION MANAGEMENT OF HOMEOSTASIS OF THE BODY ANDTHE MAIN DIRECTIONS OFTHEIR SOLUTION}

To the present day the concept of "information physiotherapy" has not been yet formulated. The scientists involved in the development of medical technology, explicated this concept differently [3]. However, the undisputed characteristic that distinguishes the informational impact from other impacts, such as energy, is intensity of used physical factor. In particular, as for the electromagnetic radiation, it should not lead to thermal heating of the body tissues. But among the information effects, the intensity of which is less than $10 \mathrm{~mW} / \mathrm{cm}^{2}$, their sources can have both natural and anthropogenic origin. Of course, such effects on living objects should have their own characteristics.

Designing and constructing of high-performance physical therapy devices require an understanding of the existing problems of information management of homeostasis of the organism and determination of the main directions in order to overcome them.

Recently, big popularity in the treatment of a wide range of human diseases has been gained by physiotherapy techniques based on the use of low-intensity electromagnetic radiation in the millimeter wave length range [2] with a flux density of energy, comparable (not exceeding $100 \mathrm{~mW} / \mathrm{cm}^{2}$ ) with background rates of anthropogenic origin.

The idea of specific impact of EMR millimeter waves on biological structures and organisms was proposed by Soviet scientists: N. D. Devyatkov, M. B. Golant and others in 1964-1965. Its main content was the following [2]. Millimeter radiation of extraterrestrial origin is strongly absorbed by the Earth's atmosphere. Therefore, the organisms could not have natural mechanisms to adapt to oscillations in the intensity of the visible range determined by external causes, but they were able to adapt to their own similar oscillations. In other words, the high sensitivity of organisms to electromagnetic radiation of millimeter wavelength range is the property of only endogenous origin. Unfortunately, this idea, which has stimulated numerous studies to establish the laws of interaction of low-intensity electromagnetic radiation from organ- isms and gave some impetus to the development of a new generation of physical therapy devices, should be recognized as only partially reflecting the diversity of the interaction of electromagnetic radiation with the organisms. It didn't allow proving the most efficient time-frequency signal structure for medicinal purposes, to establish the role of other wavelength ranges and their relationship in order to meet the challenges the correction of homeostasis, to find out the relationship of low-intensity electromagnetic radiation of natural and anthropogenic origin, and so on. The theoretical part of the research was based mainly on the totality of the proposed hypothesis resulting from the results of the experiments. Researchers supporting the above ideas, continue to search for the "best" of carriers in the millimeter wavelength range, and the most appropriate time-frequency structures for them. However, it should be recognized that in the framework of the ideas mentioned above this search a priori doomed to fail [3].

In this connection, development of a new conceptual framework for constructing a control homeostasis of the organism using EMR for medicinal purposes has become urgent. And such a framework was proposed by Russian scientists: S. N. Darovskikh and E. P. Popechitelev [4]. At its core, it is a development of ideas of Devyatkov expressed in the study of the mechanisms of interaction of organisms with EMR and its efficient use for medicinal purposes. It also reflected the well-known results of studies of the Soviet school of scientists: A. L. Chizhevsky, A. S. Presman, L. N. Gumilev, Vernadsky [5-8], etc.

The main content of the new concept is related to the evolutionary mechanisms with a high sensitivity to electromagnetic radiation caused by natural factors, both of exogenous and endogenous origin. In this case, the primary and powerful information source of the exogenous origin for organisms is the cosmic microwave background, which consists (according to the degree of priority) not only of the microwave radiation from the sun and microwave radiation of our galaxy, but also relic radiation from the center of the universe, discovered in 1965 by American scientists A. Penzias and R. Wilson. In terms of electromagnetic pollution of the environment while providing organisms with managing role of natural electromagnetic factor, it is crucial to fulfill the requirement of minimizing the excess intensity of the microwave background over human nature. Since in most cases this requirement is a priori impossible, the procedure of artificially enhancing the natural electromagnetic background or its simulated analog to the anthropogenic level has a compulsory character. The latter circumstance required for recovery of the management role of natural electromagnetic factors in organisms and property of "naturalness" of used EMR, reflect the main idea of the 
concept of counteraction to electromagnetic pollution of the environment.

A new conceptual framework allow staking into account the realities of modern electromagnetic pollution and determining the directions for the development of frequency-time structure of EMR for medical purposes and new hardware and software means for their implementation.

Two ways of implementing this concept can be suggested. The first method is associated with the modeling of the cosmic microwave radiation. The other can be realized by using special detectors of cosmic microwave background radiation with the help of space-based radio telescopes, with its subsequent retransmission (after preamplification) in the direction of the Earth Figure 2.

The basis of implementation of these methods is the use of hardware and software-hardware means to control homeostasis of the body for therapeutic purposes, which basic principles of construction are the following Figure 3 [9]:

- The use of single-channel or multi-channel wideband transmission devices in the range of natural sources microwave radiation of near and far space, providing the emission of electromagnetic energy with different types of polarization and flux density, which is comparable to the intensity of the electromagnetic background of anthropogenic origin;

- Frequency and amplitude modulation of high-frequency radiation requires using of actual parameters of low-frequency fluctuations of the cosmic microwave background or their analogs, which are formed by hardware or software means.

While determining the characteristics of the electromagnetic radiation mechanism's action on organisms in

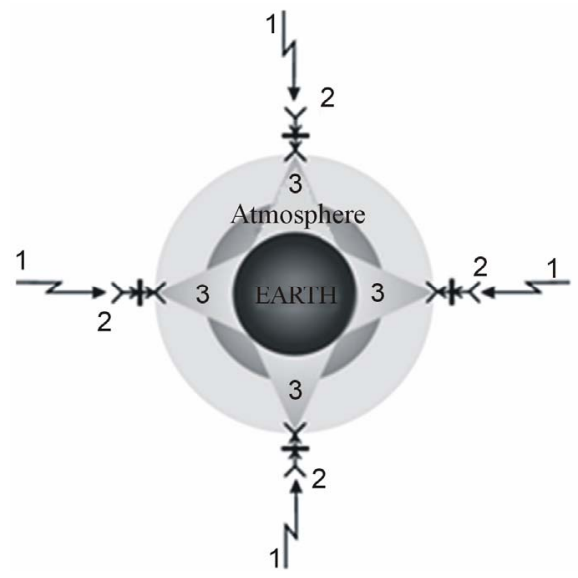

Figure 2. Explanation of a way of the organization of counteraction to electromagnetic environmental pollution: 1 -microwave space background; 2 - space radio telescopes; 3 -relaying on a surface of Earth of space radiation.

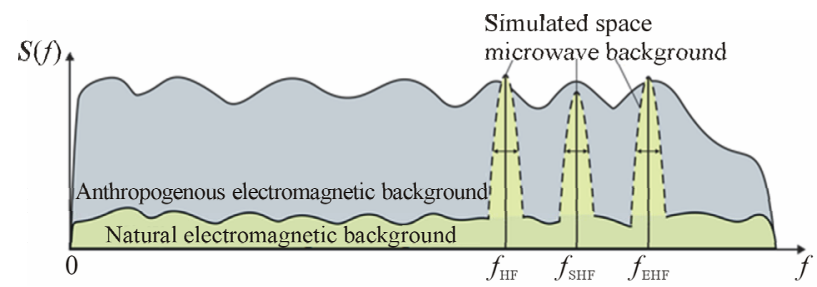

Figure 3. Explanation of the principles of creation of control units organism homeostasis.

the microwave wavelength range there should be a clear delineation of electromagnetic radiation sources: natural or anthropogenic. Without such a distinction it is not possible to carry out the systematization of the results of earlier studies. Namely for this reason at the present time there is a controversial interpretation of "good", "safety" and "dangers" of electromagnetic radiation and fields on organisms $[1,10]$. To resolve this uncertainty in modifying effect of EMR on living objects theoretical studies, which would take into account the peculiarities of their amplitude and frequency-time structure, are needed.

\section{THE THERMODYNAMIC INTERPRETATION OF THE RELATION OF INFORMATION AND ENERGY IMPACTS ON LIVING OBJECTS}

One of the unsolved problems hindering the widespread use of medical equipment for therapeutic purposes is the task of theoretical justification of relations of mechanisms of energy and information effects on living objects.

The most efficient way to solve this problem is thermodynamic approach. Using the second law of thermodynamics for open systems allows us to establish that the basis of the mechanism of information effects on living objects is a change in only free part of $\Delta F$ of internal energy $\Delta U(\Delta U=\Delta F+T \cdot \Delta S)$ of biological object without changing its associated component $\Delta E=T \cdot \Delta S$ defining change of the biological object's entropy $\Delta S$ with the help of its given thermodynamic temperature $T$.

In relation to the nature of the change of the internal energy there may be several types of information influences.

The first one is aimed at stimulating the processes leading to an increase in the capacity of a free part of the internal energy $F$ by the value $\Delta F>0$, with its subsequent use by an organism for correction of its regulatory malfunctions. One of these processes can be represented by the amplification process of the efficiency of synthesis of adenosine under hypoxic conditions of cell structures with the help of electromagnetic radiation of low intensity. Such information influence based on the use of EMR with a frequency-time structure of natural origin, is 
free from any restrictions for its use. Moreover, the higher is its efficacy, the sooner it can be applied to a biological object without any restrictions associated with the period of occurrence of a concrete disease [10,11].

This kind of exposure should be considered preferable to another approach, aiming at mobilizing the body's protective functions. In this type of exposure there is a "switching" of regulation mechanisms, the effect of which is based on the use (decrease) of the available capacity of the free energy potential $F$ in the body. The change in free energy in this case is negative $(\Delta F<0)$. The effectiveness of this type of information impact depends on the available stock of free energy in the organism. Therefore, its use for correction of regulatory functions in the body may not always lead to positive results. This type of impacts includes brief treatments using electromagnetic radiation of anthropogenic origin, which are inherently harmful to the body. The positive effect of such exposure is achieved as a result of the restructuring of the organism's functional systems in the way, when its negative effects on the body weaken. New steady state determined by such exposure, as shown by experimental studies, is often accompanied by a recovery of the patient or a significant weakening in the course of a disease. The origin of this effect is directly related to the well-known thermodynamic principle of dynamic equilibrium in nature, dubbed the "Le Chatelier-Brown's principle" [9].

There can also be a combination of considered types of information impact of on living organisms. It is based on the simultaneous effect of the physical factors on the body, some of which are aimed at increasing the free part of internal energy, and others at stimulating its protective functions associated with using of the existing free energy potential at the organism level. In this type of information influence it is important to keep a positive balance of free energy change $\Delta F$.

Energy exposures, in comparison with information influences, are directed by pronounced thermal effect not directly on the reduction of entropy $S$ of a biological object, but on its growth $\Delta S$, due to the increase in its temperature.

$$
\Delta S=S_{2}-S_{1}=\frac{M}{\mu}\left(C_{V_{\mu}} \cdot \ln \frac{T_{2}}{T_{1}}\right),
$$

where $M$-mass of the substance; $\mu$-its molar mass, $C_{V_{u}}$-the molar heat capacity at constant volume of the substance; $T_{1}$ and $T_{2}$ - the thermodynamic temperatures of the biological object respectively "before" and "after" energy exposures to it.

The purpose of this impact is to create the necessary conditions for the subsequent (after the termination of the energy exposure) reduction of the entropy by "switching" of self-synchronization within biological mechanisms. It happens due to the free part of internal energy
$\Delta F$, reported to biological system using energy exposure. The energy exposures are well studied and their use is possible only in a state of remission of a human disease.

\section{GENERAL PATTERNS OF DEVELOPMENT OF THE PATHOLOGICAL PROCESS}

Despite the well-known statement that "every disease has its own scheme of pathogenesis" there is also general law common to a wide range of human diseases [9]. It is associated with the emergence of hypoxia phenomena in tissue structures caused by various factors of both endogenous and exogenous origin, which consequently lead to disruption of homeostasis in an organism. A direct consequence of hypoxia occurring in the human body, accompanied by the displacement of the hydrogen index of the medium $(\mathrm{pH})$ in the acid side, is reduction of the efficiency of adenosine triphosphate (ATP) production, which is the main source of cell energy. It is based on the substitution of aerobic energy exchange in the cells by anaerobic. For quantitative evaluation of possible reduction of the "production" of ATP, one should compare the results of oxidation reaction (2), (3) of one mole of glucose $\left(\mathrm{C}_{6} \mathrm{H}_{12} \mathrm{O}_{6}\right)$ in case of aerobic and anaerobic cell respiration, respectively [9]

$$
\begin{gathered}
\mathrm{C}_{6} \mathrm{H}_{12} \mathrm{O}_{6}+6 \mathrm{O}_{2} \rightarrow 6 \mathrm{CO}_{2}+6 \mathrm{H}_{2} \mathrm{O}+38 \mathrm{AT} \Phi, \\
\mathrm{C}_{6} \mathrm{H}_{12} \mathrm{O}_{6} \rightarrow 2 \mathrm{CH}_{3} \mathrm{CHOHCOOH}+2 \mathrm{AT} \Phi .
\end{gathered}
$$

These reactions imply that the amount of ATP produced within aerobic respiration is 19 times greater than in case of anaerobic. The main product of anaerobic energy exchange is lactic acid $\mathrm{CH}_{3} \mathrm{CHOHCOOH}$, which "bounds much of ATP" and makes it impossible to be used in the metabolic processes to ensure homeostasis.

One of the reasons for the reduction of the effectiveness of "production" of ATP during hypoxia is the process of inactivation of the enzymes in the mitochondrial respiratory chain. In case of homeostasis reaction equation describing the interaction of negatively charged enzyme $(E n z)$ with a positively charged substrate $\left(\mathrm{SH}^{+}\right)$, has the form [9]

$$
\mathrm{Enz}^{-}+\mathrm{SH}^{+} \rightarrow \mathrm{Enz}-\mathrm{SH}
$$

Its result is the formation of the enzyme-substrate complex Enz-SH. When shifting the $\mathrm{pH}$ to the acidic side, there is a process of protonation of enzymes (its inactivation due to abundance of protons $\left(\mathrm{H}^{+}\right)$in cytosol)

$$
\mathrm{Enz}^{-}+\mathrm{H}^{+} \rightarrow \mathrm{EnzH}
$$

Thus, the development of pathological process is directly related to the significant decrease of ATP synthesized in cell structures. This decrease in efficiency of ATP synthesis underlies emergence of a deficit of the free part of inner energy $F$, which is required to maintain 
the flow of homeostatic reactions in the body.

In this regard, it is relevant to examine the physical methods aimed at restoring bioenergy potential of free energy in living objects.

\section{CHARACTERISTIC FEATURES OF RECOVERY MECHANISMS OF ORGANISM WITH A DISTURBED HOMEOSTASIS WITH THE HELP OF MICROWAVE RADIATION}

The presence of protein molecules (enzymes) involved in the synthesis of ATP, and iron ions in heme-containing groups makes it relevant to raise the question of the possibility of using magnetic resonance interactions of EMR with biological structures for solution of the problem of mitigating the negative effects caused by the process of inactivation of metalloenzymes in conditions of a $\mathrm{pH}$ shift to the acid side. The main idea of a model of MRI interactions of EMR with heme-containing proteins, which are at protonation state, is "stressing" the presence of catalytic center of these molecules by means of electromagnetic radiation on the basis of magnetic resonance. Electoral absorption of EMR by iron ions $\mathrm{Fe}^{3+}$ with a frequency equal to the Larmor precession

$$
\omega_{L}=\gamma \cdot B
$$

where $\gamma$-gyromagnetic ratio of the orbital angular moments; $B$-value of magnetic induction in hemoproteins, will lead them to an excited state, which will be accompanied by a weakening of their connection with protons. The net result of such interaction will be deprotonation of enzyme or other protein molecule with a heme-containing group in its composition.

From the standpoint of modern biomagnetism the described process of magnetic resonance interaction of heme-containing proteins with EMR, aimed at weakening the effects of hypoxia in tissue structures of the body, is not perfect. It is connected with some of unsolved fundamental problems in biomagnetism field not allowing specifying the frequency range of this interaction and its properties [10].

These shortcomings demanded the development of other model [11], which in its core is further generalization and the development of the magnetic resonance interactions model. It gave an opportunity to consider the biophysical features of the mechanisms of interaction of low intensity electromagnetic radiation (less than 10 $\mathrm{mW} / \mathrm{cm}^{2}$ ) of natural and anthropogenic origin with living organisms biological tissues and identify possible patterns of the results of this interaction that still need experimental confirmation.

It is based on assumptions about the diversity of the dispersion properties of living tissues and the presence of their resonant interactions with broadband, modulated in frequency electromagnetic radiation. This model is called "filtering model of EMR interaction with a body". According to this model, the living tissue is considered as a set of $\mathrm{N}$ filters spaced ${ }^{1}$ apart, each of which is optimally matched to the individual frequency-time variations of external EMR. This model of living tissue (filtering model) allows to consider the characteristics of the spatial distribution of its absorbed EMR energy of both anthropogenic and natural origin, as well as to make some assumptions about the possibility of emergence of several physical processes that are directly related to the result of the interaction of electromagnetic radiation with the organism. Since the pathological processes in the body usually have localized nature at different levels of organization (molecule, cell, organ, etc.), the greatest interest is connected with the examination of conditions and characteristics of the local distribution of the absorbed energy over the entire volume of tissue.

In [11] it is shown that the definition of the basic patterns of the local distribution of energy absorbed by living tissue is directly related to the study of vector function $\Psi_{s}(\Delta r, \Delta k)$, determined from the ratio

$$
\Psi_{s}(\Delta r, \Delta k)=\int_{-\infty}^{\infty} \dot{S}(r) \cdot \dot{S}^{*}(r-\Delta r) \mathrm{e}^{j \Delta k r} \mathrm{~d} r
$$

where- $\dot{S}(r)$ - the complex amplitude of the signal ${ }^{2}$, in which all the possible types of modulation are taken into account;

- $r_{c}, r_{\phi}$-the radius vectors defining the position of the signal $\dot{S}$ and the filter coordinated with it in a Cartesian coordinate system, respectively;

- $k_{c}, k_{\phi}$ - the wave vectors of the signal $\dot{S}$ and its setting in the filter, respectively; $\Delta r=r_{c}-r_{\phi}$ and $\Delta k=$ $k_{\phi}-k_{c}$.

The function $\Psi_{s}(\Delta r, \Delta k)$, which is a vector analogue of the "ambiguity function" of a radio signal, describes the spatial uncertainty $\Psi_{s}(\Delta r, \Delta k)$, in the distribution of the absorption bands of EMR by living tissue based on the availability of uncertainty of wave vector $\Delta k$. In general, the calculation of the function (7) is a rather complicated task. To evaluate the properties of the interaction of electromagnetic radiation with living tissue projection $\psi_{S x}, \psi_{S y}, \psi_{S z}$ on the axis of a Cartesian coordinate system can be used. Thus, the expression for the projection of the vector function of uncertainty $\Psi_{s}(\Delta r, \Delta k)$, on the $\mathrm{x}$-axis has the following form.

$$
\Psi(\Delta r, \Delta k)=\int_{-\infty}^{\infty} \dot{S}(x) \cdot \dot{S}^{*}(x-\Delta x) \mathrm{e}^{j \Delta k x} \mathrm{~d} x
$$

The resulting expression was used to determine the physical characteristics of interaction mechanisms of

${ }^{1}$ This property of the model reflects the existence of spatial dispersion in respect to external EMR.

2"Star" designates the complex conjugate form of expression. 
electromagnetic radiation with living tissues of both anthropogenic and natural origin [10,11].

Proposed model of the interaction of organisms with EMR implies that concentration of the absorbed energy will emerge in the area of a living tissue best matched with the time-frequency structure of the incident radiation. Thus, if the parameters of EMR of anthropogenic origin are invariable, and those of natural time-frequency structure vary from pulse to pulse, the concentration of anthropogenic electromagnetic radiation will occur in the same area of living tissue, and electromagnetic radiation of natural origin, in its various areas Figure 4.

In conditions of a weakened or ineffective thermoregulation system a process of accumulation of absorbed EMR energy of anthropogenic origin. It would be accompanied by a rise in temperature in this concrete area of living tissue. The increase in temperature in living tissue to the values of $T^{*}$ exceeding allowable limits, is a major cause of homeostasis disruption in the body with all its negative effects. This fact explains the reason why children and people with various types of mental disorders, are most exposed to anthropogenic electromagnetic radiation, even at low values of its intensity: for the first it is connected with still immature thermoregulatory system, for the second, with violation of provision of interneuron interaction not only in the implementation of thermoregulation but also other processes.

One of the important features of electromagnetic radiation of natural origin interaction with body tissues is that propagation environment may occur wave length compression process Figure 5. Underlying this phenomenon is on the one hand it is difficult to dispersive nature of the distribution, on the other hand, is complicated by the frequency of the modulated structure is used EMR. The degree of compression $K_{\text {сж }}$ (compression coefficient) can be estimated from the ratio $K_{c \varkappa}=X_{D} / X_{C}$.

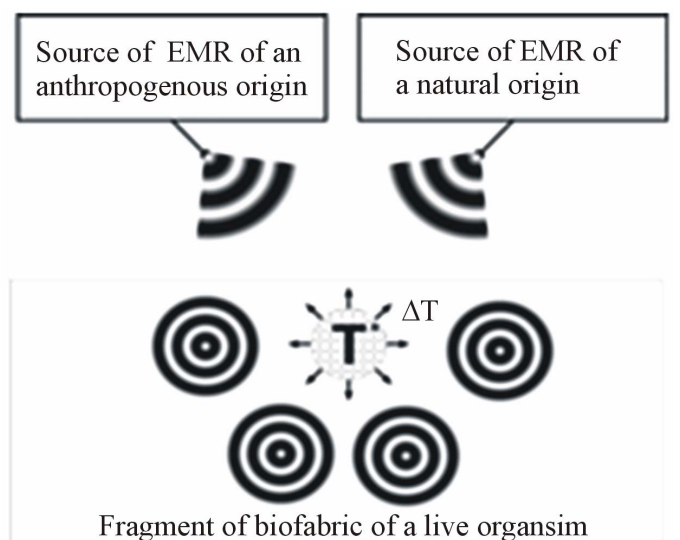

Figure 4. The explanation of distinctions in absorption of energy of EMR of an anthropogenous and natural origin.

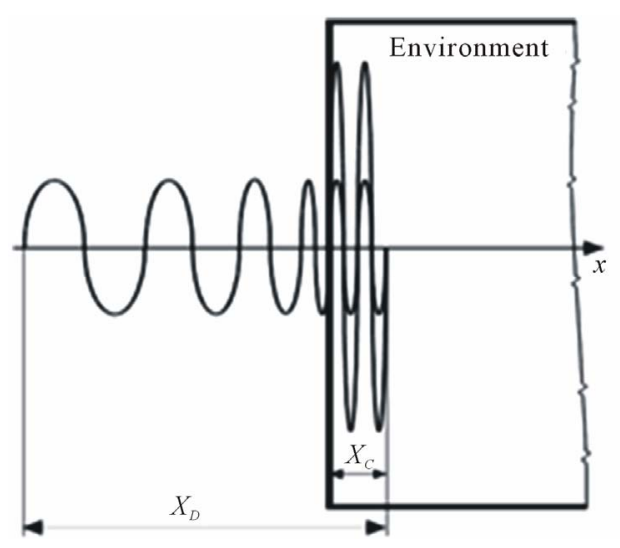

Figure 5. Explanation of the mechanism of compression of wave process.

This feature of interaction in areas of EMR absorption of natural origin leads to a $K_{с ж}$ times increase in volumetric energy density $\left(\sigma_{\varepsilon}^{*}\right)$ compared with EMR of human origin $\sigma_{\varepsilon}$, i.e. $\sigma_{\varepsilon}^{*}=K_{c \varkappa c} \sigma_{\varepsilon}$. Such concentration of energy results in an excited state of the bio molecules due to the Zeeman splitting of their energy levels. Metastable character of this state would be accompanied by the emission of electromagnetic energy at a frequency $\omega^{*}$, which would be significantly (several times) higher than the frequency $\omega$ of electromagnetic radiation affecting the living tissue.

$$
\omega^{*}=2 \pi \cdot K_{c \mathscr{K}} \cdot \sigma_{\varepsilon} \cdot V / h
$$

where $h$-Planck constant; $V$ - the amount of energy absorbed.

So during the exposure of centimeter range electromagnetic radiation on an organism, stimulated emission will lie in the millimeter wavelength range. The interaction of electromagnetic radiation with living tissue sat these frequencies is studied well. However, the fundamental difference between this radiation is connected with the fact that its origin is associated not with specific devices generating it, but with endogenous processes of EMR interactions with living tissue sat other frequencies. Apparently, if you continue to follow the model of the matched filter, the induced radiation can cause radiation at higher frequencies. This pattern can be continued and new objects (for example, water molecules), which would be affected by EMK endogenous origin can be defined. However, the foregoing is only a hypothesis, which reflects the possible evolutionary formed hierarchy of interaction of different wavelengths electromagnetic radiation with biological structures of organisms.

A part of energy of considered wave processes is also expended in places of their concentration on the excitation of elastic vibrations. They, by spreading in a medium (in Figure 4 they are represented by concentric circles), will create, in the case of electromagnetic radiation 
of natural origin usage, a complex "view" of "compression" and "thinning" and thus contribute not only to enhancing the heat transfer from the areas with high background temperature to the nearby, but also to formation of an extensive system of blood circulation in the areas of violation. The basis of this process is the emergence of the Lorentz force in the structure of biological tissue, which is described by the expression [10] for the model of an atom with an outer electron in a spherical cloud.

$$
\begin{aligned}
F_{л}= & 1 /\left(2 m\left(\omega_{o}^{2}-\omega^{2}\right)\right) \cdot\left(e^{2} \cdot K_{c \varkappa} \cdot E_{0} \cdot B_{0} \cdot \omega\right) \\
& \cdot \sin 2(\omega t-k x)
\end{aligned}
$$

where $e$-electron charge; $m$-mass of the electron; $E_{0}$, $B_{0}$ - the amplitude of the electric field and magnetic induction of electromagnetic wave respectively, $K_{c \varkappa}$ - the coefficient of length "compression" of input signal of natural origin; $\omega_{o}$ и $\omega$ - the natural frequency of the atomic electron and EMR respectively; $k$-wave number.

Pulsating nature of natural origin EMR leads to elastic oscillations in the intensity of $K_{\text {сж }}$ times higher than in the case of EMR of human origin [12].

$$
I=K_{c \mathscr{H}} \cdot\left(\Omega^{2} \cdot r \cdot X_{0}^{2} \cdot u\right) / 2
$$

where $\Omega$ - the frequency of the low-frequency electromagnetic wave amplitude pulsation,

$\rho$-density of biological tissue; $X_{0}$ - amplitude of elastic vibrations; $u$-speed of propagation of elastic oscillations.

\section{CONCLUSIONS}

Critical assessment of the conceptual foundations of previously developed physiotherapeutic millimeter-wave devices' construction helped to identify issues that need to be resolved. These problems can be solved by taking into account the nature of the exogenous electromagnetic natural factor (cosmic microwave background) within the development of the principles of control over restoring of disturbed homeostasis of the body by means of electromagnetic microwave range radiation. Discussed in the article, thermodynamic approach to the determination of the characteristics of different types of physical effects on the human body has revealed the diversity of their manifestations. This fact is particularly important with regard to information exposures and basic principles of maintaining homeostasis of the organism corresponding to them. Taking into account natural electromagnetic exogenous factor led to the development (Figure 6) of high-performance autonomous and IBM compatible devices of information physiotherapy for the prevention and treatment of a wide range of human diseases $[10,13]$.

As biomedical researches have shown, application of developed hardware has no contraindications either by the form of the disease, or the period of its occurrence
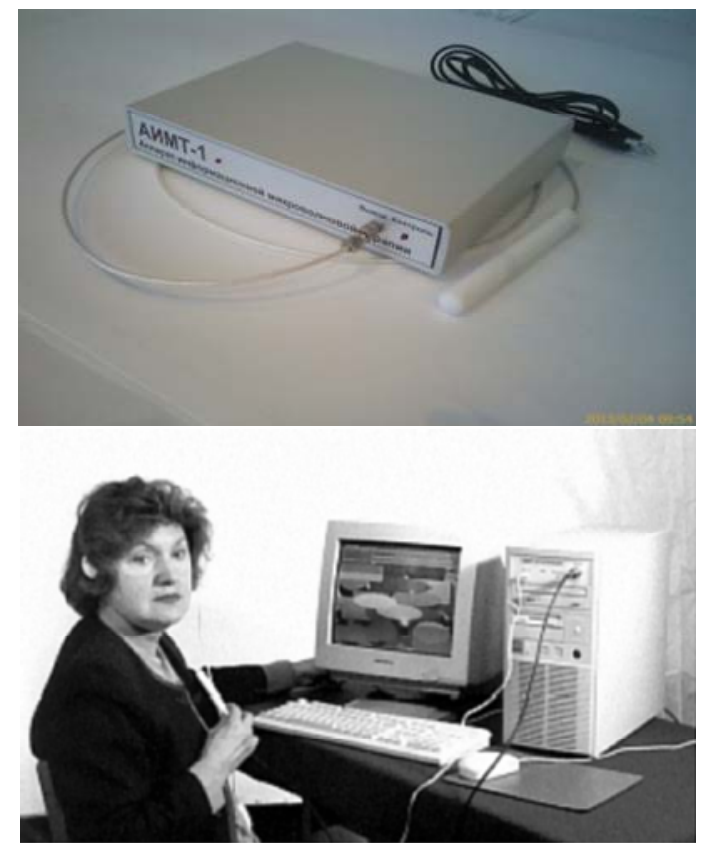

Figure 6. Devices of information physical therapy.

[14-23]. Their implementation in the system of practical public healthcare for the first time will allow putting the physical methods of correction in line with the best pharmaceutical means and giving up a large part of the existing inefficient (in the limit of potentially dangerous) physiotherapy devices and pharmaceuticals.

\section{REFERENCES}

[1] Pryahin, E.A. and Akleyev, A.V. (2007) Effect of nonionizing electromagnetic radiation on humans and animals. Academy of Medical Sciences, South Ural Scientific Center, Chelyabinsk, 219.

[2] Devyatkov, N.D., Golant, M.B. and Betsky, O.V. (1991) Millimeter waves and their role in life processes. Radio and communication. 186.

[3] Darovskikh, S.N. (2012) The problems of information control over homeostasis of the organism by means of electromagnetic radiation of millimeter range and the main directions of their solutions. Biomedical Radio Electronics, 3, 3-10.

[4] Darovskih, S.N. and Popechitelev, E.P. (2012) Some aspects of the mechanisms of information physiotherapy. Proceedings of the SPSETU “LETT", SPSETU "LETI”, St. Petersburg, 2, 81-88.

[5] Chizhevsky, A.L. (1973) Earthly echo of solar storms. 347.

[6] Presman, A.S. (1974) Electromagnetic alarm in nature. Facts, hypotheses, ways of research. Sov. Radio, Moscow, 64.

[7] Gumilev, L.N. (2002) Ethno genesis and the biosphere of the earth. SZKEO, Ltd., Editor House "Crystal", St. Petersburg, 639. 
[8] Vernadsky, V.I. (1994) Living matter and the biosphere. Nauka, Moscow, 672.

[9] Grin, N., Stout, W. and Taylor, D. (1993) Biology. Moscow, 327-374.

[10] Darovskikh, S.N. and Popechitelev, E.P. (2012) Modern aspects of building information electromagnetic therapy devices. Saarbruecken Publishing House LAP LAMBERT, 241.

[11] Darovskikh, S.N. (2011) Mathematical model of information interaction of natural objects with electromagnetic radiation. Bulletin of the South Ural State University, "Computer Technology, Management, Electronics," Publishing Center SUSU, Chelyabinsk, 219, 45-48.

[12] Orir, J. (1981) Physics. Springer-Verlag, 2, 622.

[13] Popechitelev, E.P. and Darovskikh, S.N. (2011) Information physiotherapy and hardware means of its implementation. Proceedings of the Southwestern State University, "Management, Computer Engineering, Computer Science", Publishing Center SWSU, Kursk, 60-66.

[14] Darovskikh, S.N. and Razzhivin, A.A. (1996) Information-wave methods of correcting violations of regulatory functions in living organisms. Foreign Electronics, 12, 33-40.

[15] Darovskikh, S.N., Tolstykh, E.I., Razzhivin, A.A., et al. (1999) Research of modifying exposure of electromagnetic radiation of low intensity together of its interaction with ionizing energy flows on biological objects. Biomedical Electronics, 8, 31-35.

[16] Darovskikh, S.N., Uzunova, A.N., Boytsov, V.M. and Razzhivin, A.A. (2002) Some aspects of the informational approach in physical therapy. Biomedical Technology and Electronics, 12, 27-32.

[17] Darovskikh, S.N., Rassokhin, A.G. and Kuznetsov, M.E.
(2005) The management role of the relic radiation from the center of the universe in wildlife. Biomedical Technology and Electronics, 6, 40-45.

[18] Darovskikh, S.N., Erofeev, S.A., Chikorina, N.K., et al. (2006) Experience of microwave magnetic resonance therapy implementation in an experiment with a dog leg lengthening. Genius of Orthopaedics: Scientific Journal, 1, 48-51.

[19] Uzunova, A.N., Zaitseva, M.L., Darovskikh, S.N., Ryabov, N.D. and Koptyaeva, N.V. (1995) The effectiveness of microwave magnetic resonance therapy for obstructive bronchitis among children. Pediatrics, 5, 44-45.

[20] Uzunova, A.N., Kurilova, E.V., Darovskikh, S.N. and Kozlovskaya, N.A. (1997) Microwave therapy in the complex treatment of chronic secondary pyelonephritis among children. Issues of Baleological Physical Therapy, 3, 2728.

[21] Uzunova, A.N., Kurilova, E.V. and Darovskikh, S.N. (2004) The influence of microwave magnetic resonance therapy on certain factors of the local immune defense of the respiratory tract of sickly children. Issues of Baleology, Physical Therapy and Therapeutic Physical Culture, 6, 2729.

[22] Uzunova, A.N., Kofanov, R.V. and Chernysh, N.N. (2005) Microwave therapy in the complex rehabilitation of children suffering from chronic sensorneural baryecoia. Issues of Balneology, Physical Therapy and Therapeutic Physical Culture, 4, 28-30.

[23] Uzunov, A.N., Gorlova, N.V. and Darovskikh, S.N. (2006) Usage of microwave magnetic resonance physiotherapy device for correction of metabolic disorders among children with pneumonia. Bulletin of the South Ural State University. Education, Health, Physical Education, 1-3, 252-255. 